\title{
Asymmetric features of short-term blood pressure variability
}

\author{
Przemyslaw Guzik ${ }^{1}$, Jaroslaw Piskorski ${ }^{2}$, Tomasz Krauze ${ }^{1}$, Krzysztof Narkiewicz $^{3}$, Andrzej Wykretowicz ${ }^{1}$ \\ and Henryk Wysocki ${ }^{1}$
}

Prolongations of cardiac cycles have a significantly larger contribution to short-term heart rate variability than shorteningsthis is called heart rate asymmetry. Our aim is to establish the existence of blood pressure asymmetry phenomenon, which has not been done so far. We used 30-min resting continuous recordings of finger pressure waveforms from 227 healthy young volunteers (19-31 years old; 97 female), and performed Poincaré plot analysis of systolic blood pressure (SBP) to quantify the effect. Median contribution of SBP increases $\left(C_{i}\right)$ to short-term blood pressure variability was $52.8 \%$ (inter-quartile range: $50.9-55.1 \%)$ and median number of SBP increases $\left(N_{i}\right)$ was $48.8 \%$ (inter-quartile range: $47.2-50.1 \%$ ). The $C_{i}>50 \%$ was found in $82 \%(P<0.0001$; binomial test $)$ and $N_{i}<50 \%$ in $75 \%(P<0.0001)$ of the subjects. Although SBP increases are significantly less abundant than reductions, their contribution to short-term blood pressure variability is significantly larger, which means that short-term blood pressure variability is asymmetric. SBP increases and reductions have unequal contribution to short-term blood pressure variability at supine rest in young healthy people. As this asymmetric behavior of blood pressure variability is present in most of the healthy studied people at rest, it can be concluded that blood pressure asymmetry is a physiological phenomenon.

Hypertension Research (2010) 33, 1199-1205; doi:10.1038/hr.2010.138; published online 5 August 2010

Keywords: blood pressure asymmetry; blood pressure variability; heart rate asymmetry; heart rate variability; sympatheticparasympathetic balance

\section{INTRODUCTION}

Both heart rate variability (HRV) and blood pressure variability (BPV) are related to autonomic activity and provide some information on the mechanisms regulating the cardiovascular system. ${ }^{1-6}$ Various physiological oscillations (for example, respiration, Mayer waves) and cardiovascular reflexes (for example, arterial and cardiopulmonary baroreflexes) are responsible for HRV and BPV. ${ }^{1-4,6-9}$ A number of mathematical approaches like the time-domain analysis, spectral analysis, graphic or nonlinear methods have been applied in the analysis of HRV and BPV. ${ }^{1-6,9-13}$ Even though heart rate and blood pressure are different cardiovascular signals, very often the same techniques are used for the quantification of both HRV and BPV. ${ }^{1,3-9,13}$ On the other hand, descriptors of baroreflex function like baroreflex sensitivity and baroreflex delay (the delay of sinus node response to a change in blood pressure) reflect the blood pressure influence on heart rate more directly. $3,9,14$

Most of the methods applied to the analysis of HRV and BPV look at the changes of the duration of cardiac cycle of sinus origin (RR) intervals or blood pressure, but do not analyze their direction, that is, whether they represent increases or decreases of heart rate or blood pressure, respectively. ${ }^{1-11,13}$ Recently, however, some methods, which allow separate analysis of decelerations and accelerations of heart rate or increases (i) and reductions ( $r$ ) in blood pressure, have been described. ${ }^{12,15,16}$ Among them are such techniques like phase-rectified signal averaging or variance partitioning by Poincaré plot. ${ }^{12,15,16}$

The Poincare plot analysis of RR intervals was used to show the existence of the physiological phenomenon of heart rate asymmetry in young healthy individuals. ${ }^{12,16}$ At supine rest, the prolongations of cardiac cycles usually have a significantly larger contribution to shortterm HRV than shortenings, and this unidirectional phenomenon is present in the majority $(>80 \%)$ of electrocardiography (ECG) recordings. ${ }^{12,16}$ Porta et al. ${ }^{17}$ have also shown that the number of prolongations of RR intervals is significantly smaller than the number of shortenings.

Baroreflex function is known to show some asymmetric properties, for example, baroreflex sensitivity calculated for blood pressure increases and prolongations of RR intervals after phenylephrine infusion or neck suction is different from that calculated for blood pressure reductions, and shortenings of cardiac cycles after nitroprusside or the application of neck pressure..$^{9,18-21}$ As both HRV and

${ }^{1}$ Department of Cardiology-Intensive Therapy, University of Medical Sciences in Poznan, Poznan, Poland; 2Institute of Physics, University of Zielona Gora, Zielona Gora, Poland and ${ }^{3}$ Department of Hypertension and Diabetology, Medical University of Gdansk, Gdansk, Poland

Correspondence: Dr P Guzik, Department of Cardiology-Intensive Therapy, University School of Medicine in Poznan, 49 Przybyszewskiego Str, Poznan 60-355, Poland.

E-mail: pguzik@ptkardio.pl

A part of the results was presented during the 5th Conference of the European Study Group on Cardiovascular Oscillations in Parma, Italy on 7-9 of April 2008 and during the 19th European Meeting on Hypertension in Milan on 12-16 of June 2009.

Received 27 November 2009; revised 3 June 2010; accepted 25 June 2010; published online 5 August 2010 
baroreflex function are asymmetric, it can be expected that a similar phenomenon may be found in BPV. The primary aim of this study is to establish the existence of asymmetric features of short-term blood pressure asymmetry, in short, blood pressure asymmetry, by separately analyzing the information from systolic blood pressure (SBP) increases and reductions. The secondary aim is to look for any association between measures of blood pressure asymmetry and indirect indices of autonomic modulation of the cardiovascular system, namely BPV, HRV, heart rate asymmetry and baroreflex function.

\section{METHODS}

\section{Subjects}

In total, 227 healthy volunteers (19-31 years old) were enrolled. There were 97 female participants in the studied group. Their health status was evaluated by history, routine cardiac physical examination with anthropometric measurements and resting standard 12-lead ECG. All subjects had to fulfill the following inclusion criteria: brachial blood pressure $<140 / 90 \mathrm{~mm} \mathrm{Hg}$ normal ECG with sinus rhythm $<100 / \mathrm{min}$, no drug or alcohol addiction, no use of medications or hormones in the past 3 months, no involvement in endurance training and sports. Brachial blood pressure was measured in the sitting position after $10 \mathrm{~min}$ rest, with an oscillometric method (Omron M-5; Omron Healthcare Ltd., Milton Keynes, UK) before the ECG and continuous finger blood pressure recording. The following anthropometrics were used in the study: body height and weight, body mass index and waist-to-hip ratio. All subjects refrained from alcohol for at least $24 \mathrm{~h}$, and tobacco and coffee for at least $12 \mathrm{~h}$ before the study. All volunteers gave informed consent to participate in the study. Both the informed consent form and the project were approved by the University Bioethical Committee.

Non-invasive continuous finger blood pressure and ECG recording After allowing $15 \mathrm{~min}$ for cardiovascular adaptation to the supine position, resting signals of non-invasive pressure waveform (Portapres 2, FMS, Amsterdam, The Netherlands) and bipolar chest ECG (Porti 5, TMSI, Oldenzaal, The Netherlands) were recorded for $30 \mathrm{~min}$. With the use of the libRASCH/RASCHlab software from the libRASCH project (version. 0.6.1; http://www.librasch.org, Munich, Germany), an automatic evaluation of ECG was preliminarily performed and then it was followed by a visual inspection of all beats and the introduction of the necessary corrections to the obtained values. ${ }^{22}$ The values of SBP, diastolic and mean blood pressure measured at finger artery, as well as RR intervals, that is, duration of each cardiac cycle with appropriate beat type annotation (normal, supraventricular or ventricular) were retrieved from stored recordings. In further analysis, only SBP and RR interval values related to beats of sinus origin were used.

\section{Blood pressure asymmetry by the Poincare plot analysis of SBP}

The Poincare plot is a graphical representation of temporal correlations within the SBP values, and it is similar to the Poincare plot analysis of RR intervals derived from the ECG. ${ }^{10-13,16,23}$ In this plot for SBP (Figure 1), each SBP is a function of the preceding SBP, that is, the value of SBP of the current cardiac beat $\left(\mathrm{SBP}_{\mathrm{n}}\right)$ is represented on the $x$-axis, and the value of SBP of the next beat $\left(\mathrm{SBP}_{\mathrm{n}+1}\right)$ on the $y$-axis. In this way each point $\left(\mathrm{SBP}_{\mathrm{n}}, \mathrm{SBP}_{\mathrm{n}+1}\right)$ in the plot corresponds to two successive SBP values. The following descriptors of Poincaré plots of SBP were calculated with in-house software written in Python (Python Software Foundation, www.python.org, Wolfeboro Falls, NH, USA): ${ }^{10-13,16,23}$

- $\mathrm{SD} 1^{2}$ - the square of the s.d., or variance, quantifying the dispersion of all points in the plot across the identity line-this parameter is a measure of short-term SBP variance $\left(\mathrm{SD}^{2}\right)$, that is, the short-term BPV, and according to the variance properties, $\mathrm{SD} 1^{2}$ can be decomposed into two parts, that is, $\mathrm{SD} 1_{i}^{2}$ and $\mathrm{SD}_{r}{ }^{2}$ :

- $\mathrm{SD} 1_{i}^{2}$ - the part of $\mathrm{SD}^{2}$ contributed by the points in the Poincare plot above the identity line-these points correspond only to SBP increases.

- $\mathrm{SD}_{r}{ }^{2}$ - the part of $\mathrm{SD}^{2}$ contributed by the points in the Poincare plot below the identity line-these points correspond only to SBP reductions.

$\mathrm{SD}^{2}$ for SBP is used only for $\mathrm{Cl}_{i}$ and $\mathrm{Cl}_{r}$ quantification and is not shown in the Results section.

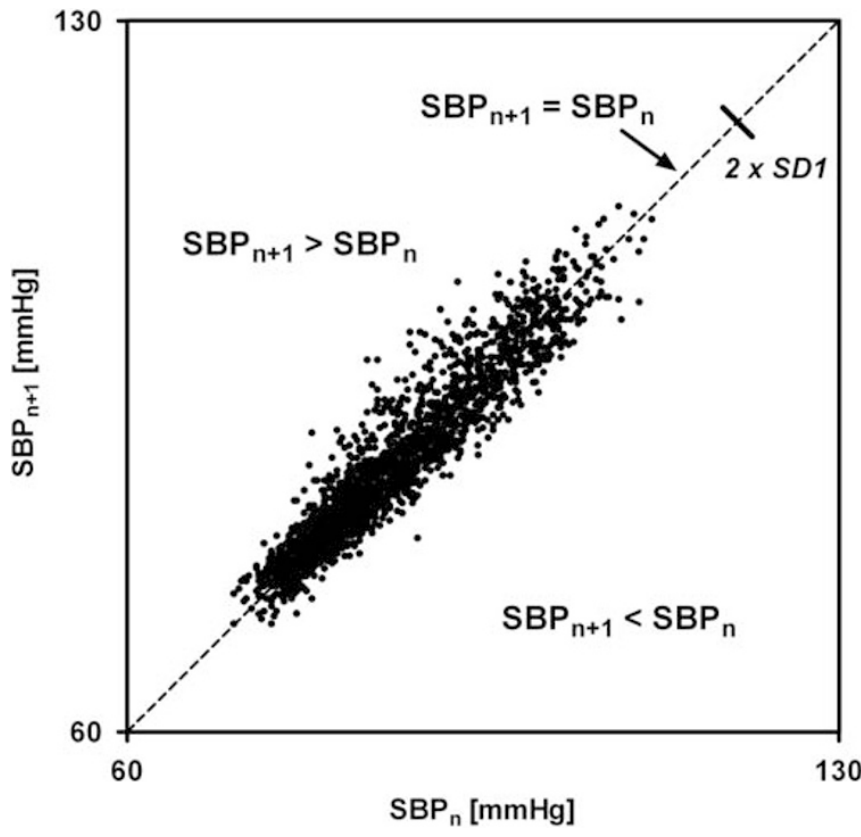

Figure $1 \mathrm{~A}$ sample Poincaré plot analysis of an SBP time series derived from a 30-min resting recording of finger pressure waveform from a healthy subject. The dashed line is the identity line; all points on this line correspond to two neighboring beats of equal values of $\mathrm{SBP}\left(\mathrm{SBP}_{\mathrm{n}+1}=\mathrm{SBP}_{\mathrm{n}}\right)$; all points above this line correspond to SBP increases $\left(\mathrm{SBP}_{n+1}>\mathrm{SBP}_{n}\right)$; all points below this line correspond to $\mathrm{SBP}$ reductions $\left(\mathrm{SBP}_{\mathrm{n}+1}<\mathrm{SBP}_{\mathrm{n}}\right)$. SD1 is the square root of $\mathrm{SD}^{2}$ and it is a measure of short-term blood pressure variability. SBP, systolic blood pressure; $\mathrm{SBP}_{n}, \mathrm{SBP}$ of the current cardiac beat; $\mathrm{SBP}_{\mathrm{n}+1}$, SBP of the next beat; SD1, the standard deviation quantifying the dispersion of points in the Poincaré plot across the identity line and short-term variance of SBP.

All neighboring cardiac beats with equal SBP values are localized on the identity line. These points do not generate any short-term variance, and thus do not contribute to $\mathrm{SD}^{2}$

Because $\mathrm{SD}^{2}=\mathrm{SD} 1_{i}{ }^{2}+\mathrm{SD}_{r}{ }^{2}$, both $\mathrm{SD}_{i}{ }^{2}$ and $\mathrm{SD}_{r}{ }^{2}$ are approximately proportional to $\mathrm{SD}^{2}$ and as such behave accordingly. ${ }^{12,16}$ When $\mathrm{SD}^{2}$ is large, then its parts have large values too, when $\mathrm{SD} 1^{2}$ is reduced then $\mathrm{SD} 1_{i}{ }^{2}$ and $\mathrm{SD} 1_{r}{ }^{2}$ are decreased as well.

There is a large inter-personal variability in BPV. For this reason, we use the $\mathrm{Cl}_{i}$ and $\mathrm{Cl}_{r}$, which are normalized to the individual's BPV variance and still reflect the contributions of SBP increases and reductions to short-term BPV, respectively. This approach follows the methodology developed and used for heart rate asymmetry. ${ }^{12,16}$

$$
\begin{aligned}
& \mathrm{C}_{i}[\%]=\mathrm{SD}_{i}^{2} / \mathrm{SD}^{2} \times 100, \\
& \mathrm{C}_{r}[\%]=\mathrm{SD}_{r}^{2} / \mathrm{SD}^{2} \times 100 .
\end{aligned}
$$

$\mathrm{Cl}_{i}$ describes the relative contribution of $\mathrm{SBP}$ increases and $\mathrm{Cl}_{r}$ shows the relative contribution of SBP reductions to the short-term BPV. The values of $\mathrm{Cl}_{i}$ and $\mathrm{Cl}_{r}$ change in the range between 0 and $100 \%$ and they sum to $100 \%$. For this reason, to avoid redundancy, only $\mathrm{Cl}_{i}$ will be shown in the results section.

Two other descriptors of blood pressure asymmetry are based on the analysis of the number of SBP increases $\left(n_{i}\right)$ and reductions $\left(n_{r}\right)$ corresponding to the numbers of points above and below the identity line of the Poincare plot, respectively. ${ }^{17,24}$ However, as both $n_{i}$ and $n_{r}$ are strictly dependent on the length of the recording, in further analysis these numbers are also normalized:

$$
\begin{aligned}
& N_{\mathrm{i}}[\%]=n_{\mathrm{i}} /\left(n_{\mathrm{i}}+n_{\mathrm{r}}\right) \times 100 \\
& N_{\mathrm{r}}[\%]=n_{\mathrm{r}} /\left(n_{\mathrm{i}}+n_{\mathrm{r}}\right) \times 100
\end{aligned}
$$


In this way, the $N_{i}$ describes the relative number of SBP increases and $N_{r}$ shows the relative number of SBP reductions to all the changes in SBP. The values of $N_{i}$ and $N_{r}$ change in the range between 0 and $100 \%$ and sum to $100 \%$. For this reason, to avoid redundancy, only $N_{i}$ will be shown in the results section.

The approach presented above is one of many, and we believe it is very general. Other approaches exist, like for example, the approach, which treats the cardiovascular time series as a representation of a multidimensional object for which a phase space, and consequently, embedding space exist. ${ }^{24-26}$ In this approach it is possible to go further and analyze asymmetries at higher dimensions. This approach, albeit interesting and very promising, makes a number of assumptions and uses quite involved mathematics-for a more comprehensive review see. ${ }^{24}$ We believe that it is important to first establish the phenomenon with a readily understandable approach and then, possibly, move on to more advanced methodology.

\section{Blood pressure variability}

The mean SBP was used to describe the average of the SBP time series. For the total variance of SBP, the s.d. of SBP values for all normal beats was used. For the analysis of the SBP variance at frequency range $0.04-0.15 \mathrm{~Hz}$ and $0.15-0.4 \mathrm{~Hz}$, low frequency power (LFBP) and high frequency power (HFBP), respectively, was calculated with the use of Lomb periodograms for spectral analysis. ${ }^{3-9,27-30}$

\section{Heart rate variability}

For the RR interval time series we used a similar approach as for the SBP signal, that is, the mean RR interval, s.d. of all normal RR intervals for total HRV and the results of spectral analysis by Lomb periodograms. ${ }^{1,2,9,28,29}$ However, for spectral HRV analysis, in addition to the low frequency power (LFRR; 0.04$0.15 \mathrm{~Hz}$ ) and high frequency power (HFRR; $0.15-0.4 \mathrm{~Hz}$ ), two other variables were calculated. First, LFRR was also expressed in normalized units $\left(\operatorname{LFRR}_{\mathrm{nu}}\right)$, which is the ratio of LFRR to total power of RR intervals $(0.00-0.4 \mathrm{~Hz})$ diminished by the power of very low frequency of RR intervals $(0.00-0.04 \mathrm{~Hz})$. Second, the LFRR to HFRR ratio (LFRR/HFRR) was also applied. ${ }^{1-3,9,27}$ The last two variables were only used for HRV and not for BPV analysis. For the LFBP there is no parasympathetic influence, and thus LFBP is a better sympathetic index than LFRR. The RR interval LFBP reflects complex sympathetic and parasympathetic effects, and thus LFRR $_{\text {nu }}$ and LFRR/HFRR are not only commonly used, but are among those recommended. ${ }^{1}$

\section{Heart rate asymmetry}

For heart rate asymmetry the following previously reported variables were calculated with the use of Poincare plot analysis of RR intervals and in-house software written in Python (Python Foundation, USA): ${ }^{12,16,17}$

- $\mathrm{SD}^{2}$ - the variance quantifying the dispersion of all points in the plot across the identity line-this is the measure of the short-term HRV and is used only for $\mathrm{Cl}_{d}$ and $\mathrm{Cl}_{a}$ quantification, see below;

- $\mathrm{SD} 1_{d}^{2}$-the part of $\mathrm{SD}^{2}$ contributed by the points in the Poincare plot above the identity line-these points correspond only to decelerations of heart rate (prolongations of RR intervals). ${ }^{12,16}$

- $\mathrm{SD} 1_{a}^{2}$-the part of $\mathrm{SD}^{2}$ contributed by the points in the Poincare plot below the identity line-these points correspond only to accelerations of heart rate (shortenings of RR intervals). ${ }^{12,16}$

- $\mathrm{Cl}_{d}$ and $\mathrm{Cl}_{a}$, are the ratios of $\mathrm{SD} 1_{d}^{2}$ to $\mathrm{SD}^{2}$, and $\mathrm{SD}_{a}{ }^{2}$ to $\mathrm{SD} 1^{2}$, respectively, and show the contributions of heart rate decelerations and accelerations to short-term $\mathrm{HRV}$, respectively. $\mathrm{As} \mathrm{Cl}_{d}+\mathrm{Cl}_{a}=100 \%$, thus to avoid redundancy, only $\mathrm{Cl}_{d}$ will be shown in the Results section.

- $N_{d}$, called also Porta's index, is the ratio of the number of heart rate decelerations to the number of all changing cardiac cycles, that is, the total number of all decelerations and accelerations of the heart rate. ${ }^{17,24}$ Analogously, $N_{a}$ can be calculated for heart rate accelerations; however, $N_{d}+N_{a}$ equals $100 \%$, and therefore to avoid redundancy, only $N_{d}$ will be shown in the results section.

\section{Spontaneous baroreflex function}

Spontaneous baroreflex function was quantified with the use of the crosscorrelation method, which is a development of the sequential method. ${ }^{14}$
This method gives two independent descriptors of the baroreflex function, that is, baroreflex sensitivity and baroreflex delay, which is the average time of sinus node response to a change in SBP.

\section{Statistical analysis}

The descriptors introduced for blood pressure asymmetry give us two types of information. The first information is about the magnitude of the effect, that is by how much the contributions from SBP reductions are larger than those of SBP increases. The other type is the frequency of the effect, that is, the proportion of subjects in whom the postulated blood pressure asymmetry is observed.

Because of the non-normal distribution of blood pressure asymmetry descriptors (established by the Shapiro-Wilk test) all continuous data are presented in tables as median and inter-quartile range, and the non-parametric Spearman correlation was used for the analysis of the association between measures of blood pressure asymmetry and continuous clinical characteristics or other indices of autonomic modulation. The presence of blood pressure asymmetry as a unidirectional phenomenon was recorded in a subject when $\mathrm{Cl}_{i}>50 \%$ or $N_{i}<50 \%$. Similarly, the presence of heart rate asymmetry as a unidirectional phenomenon was defined when $\mathrm{Cl}_{d}>50 \%$ or $\mathrm{N}_{d}<50 \%$. The binomial test was applied to establish whether the proportion of recordings with $\mathrm{Cl}_{i}>50 \%$ or $\mathrm{N}_{i}<50 \%$ for blood pressure asymmetry and $\mathrm{Cl}_{d}>50 \%$ or $N_{d}<50 \%$ for heart rate asymmetry is different from 0.5 . For the evaluation of the co-existence of blood pressure asymmetry with heart rate asymmetry, contingency tables with observed and predicted values for the following pairs $\mathrm{Cl}_{i} \& N_{i}, \mathrm{Cl}_{i} \& \mathrm{Cl}_{d}, \mathrm{Cl}_{d} \& N_{d}$, and $N_{i} \& N_{d}$ were analyzed. Pearson's $\chi^{2}$ variable was used to compare the frequencies expected under independence with those obtained in the studied sample. In addition, the relationship between gender and blood pressure asymmetry indices was quantified with the non-parametric Mann-Whitney $U$ test and Fisher's exact test. Values only with $P<0.05$ were considered statistically significant. Note also that the power and sample size considerations described in ${ }^{12}$ are of general nature and apply to the this study as well. All statistical analyses were done with the R statistical system (version 2.71; The R Foundation for Statistical Computing, www.r-project.org, Vienna, Austria) and Prism (version 5.01, GraphPad, San Diego, CA, USA)

\section{RESULTS}

\section{Baseline characteristics}

There were 97 female participants in the studied group. The mean values of anthropometrics, resting heart rate and blood pressure profile were within the normal range in the studied group (Table 1).

\section{Blood pressure asymmetry and indirect measures of autonomic modulation of the cardiovascular system}

Table 1 shows also the magnitude statistics for mean SBP, descriptors of blood pressure asymmetry and blood pressure variability of SBP, mean RR interval, heart rate variability and heart rate asymmetry, as well as measures of baroreflex function.

\section{Relation of blood pressure asymmetry to clinical characteristics} and autonomic modulation of the cardiovascular system Table 2 shows results of the Spearman correlation between the measures of blood pressure asymmetry for SBP and clinical characteristics or mean finger SBP, measures of BPV for SBP, mean RR interval, HRV, heart rate asymmetry and descriptors of baroreflex function.

Although the age of the studied volunteers was quite narrow, that is, 12 years (19-31 years old), there was a weak, but significant correlation between blood pressure asymmetry and age. There were no significant correlations between blood pressure asymmetry measures and anthropometrics of studied individuals, resting heart rate (sitting position during measurement of brachial blood pressure before 30-min recording in supine position was started), or brachial pulse pressure. For the remaining clinical characteristics there were weak correlations between brachial systolic blood pressure $\mathrm{SD} 1_{r}^{2}$ or brachial diastolic pressure and $\mathrm{Cl}_{i}$ and $N_{i}$. 
Table 1 Clinical characteristics and results of the analysis of blood pressure asymmetry and BPV for systolic blood pressure, HRV, heart rate asymmetry and baroreflex function in 30-min resting recordings from healthy volunteers

\begin{tabular}{|c|c|c|}
\hline & Median & $I Q R$ \\
\hline \multicolumn{3}{|l|}{ Clinical characteristics } \\
\hline Age (years) & 23.0 & $22.0-25.0$ \\
\hline Height (cm) & 174 & $168-182$ \\
\hline Weight (kg) & 68 & $57-77$ \\
\hline Body mass index $\left(\mathrm{kg} \mathrm{m}^{-2}\right)$ & 21.9 & $20.0-24.0$ \\
\hline Waist-to-hip ratio $\left(\mathrm{cm} \mathrm{cm}^{-1}\right)$ & 0.8 & $0.7-0.9$ \\
\hline Resting heart rate (beats/min) & 66.7 & $61.3-73.6$ \\
\hline Brachial systolic blood pressure ( $\mathrm{mm} \mathrm{Hg}$ ) & 112.5 & $105.5-120.7$ \\
\hline Brachial diastolic blood pressure $(\mathrm{mm} \mathrm{Hg})$ & 67.0 & $61.2-72.4$ \\
\hline Brachial pulse pressure $(\mathrm{mm} \mathrm{Hg})$ & 46 & $40-52.6$ \\
\hline \multicolumn{3}{|l|}{ SBP time series } \\
\hline Mean SBP $\left(\mathrm{mm} \mathrm{Hg}^{-2}\right)$ & 117.5 & 105.7-130.9 \\
\hline $\mathrm{SD} 1_{i}^{2}\left(\mathrm{~mm} \mathrm{Hg}^{-2}\right)$ & 2.3 & $1.6-3.7$ \\
\hline $\mathrm{SD} 1_{r}^{2}(\mathrm{~mm} \mathrm{Hg})$ & 2.0 & $1.4-3.3$ \\
\hline $\mathrm{C} 1_{i}(\%)$ & 52.8 & $50.9-55.1$ \\
\hline$N_{i}(\%)$ & 48.8 & $47.2-50.1$ \\
\hline $\mathrm{SDBP}(\mathrm{mm} \mathrm{Hg})$ & 7.7 & $6.2-9.4$ \\
\hline LFBP $\left(\mathrm{mm} \mathrm{Hg}^{2}\right)$ & 9.7 & $6.5-15$ \\
\hline $\mathrm{HFBP}\left(\mathrm{mm} \mathrm{Hg} \mathrm{H}^{2}\right)$ & 4.1 & $2.7-6.4$ \\
\hline \multicolumn{3}{|l|}{$R R$ interval time series } \\
\hline Mean RR interval (ms) & 884.7 & 782.3-949.4 \\
\hline SDNN (ms) & 63.9 & $51.7-81.3$ \\
\hline $\operatorname{LFRR}\left(\mathrm{ms}^{2}\right)$ & 1119.5 & 740.9-2043.6 \\
\hline $\operatorname{HFRR}\left(\mathrm{ms}^{2}\right)$ & 907.5 & 530.9-1935.6 \\
\hline $\mathrm{LFRR}_{\text {nu }}$ & 56.2 & $46.1-63$ \\
\hline LFRR/HFRR & 1.3 & $0.9-1.7$ \\
\hline $\mathrm{SD} 1 d^{2}\left(\mathrm{~ms}^{2}\right)$ & 601.8 & $310.2-1354.4$ \\
\hline $\mathrm{SD} 1_{a}^{2}\left(\mathrm{~ms}^{2}\right)$ & 497.5 & 287.5-1051.6 \\
\hline $\mathrm{C} 1_{d}(\%)$ & 54.4 & $51.2-58.4$ \\
\hline$N_{d}(\%)$ & 49.3 & $47.6-51$ \\
\hline \multicolumn{3}{|l|}{ Baroreflex } \\
\hline $\mathrm{BRS}\left(\mathrm{ms} \mathrm{mm} \mathrm{Hg}^{-1}\right.$ ) & 14.7 & $11.1-19.9$ \\
\hline Baroreflex delay (s) & 1.9 & $1.7-2.2$ \\
\hline
\end{tabular}

Abbreviations: BPV, blood pressure variability; BRS, baroreflex sensitivity; $\mathrm{C} 1_{d}$, relative contributions of heart rate decelerations to short-term HRV; $\mathrm{C} 1_{\text {i }}$, relative contributions of SBP increases to short-term BPV; HFBP, power of high frequency oscillations of SBP; HFRR, power of high frequency oscillations of RR intervals; HRV, heart rate variability; IQR, inter-quartile range; LFBP, power of low frequency oscillations of SBP; LFRR, power of low frequency oscillations of RR intervals; LFRR/HFRR, the ratio of LFRR to HFRR; LFRR $_{\text {nu }}$, power of low frequency oscillations of RR intervals in normalized units; $N_{d}$, the relative number of heart rate decelerations to the number of all changing RR intervals; $N_{i}$, the relative number of SBP increases to the number of all changing values of SBP; RR interval, duration of cardiac cycle of sinus origin; SBP, systolic blood pressure; $S D 1_{a}^{2}$, the part of short-term HRV derived from heart rate accelerations; SD1 $d^{2}$, the part of short-term HRV derived from heart rate decelerations; rate accelerations; $\mathrm{SD}_{d^{2}}{ }^{2}$, the part of short-term HRV derived from heart rate decelerations; short-term variance derived from SBP reductions; SDBP, the standard deviation of SBP values for all normal beats; SDNN, standard deviation of all normal RR intervals.

The correlation between mean finger SBP and measures of blood pressure asymmetry was significant, but weak. The correlation between $\mathrm{SD} 1_{i}{ }^{2}$ or $\mathrm{SD} 1_{r}{ }^{2}$ and measures of BPV was significant and moderate for SBP values for all normal beats (measure of total variance) and LFBP, but it was strong for the high frequency oscillations power $(r=0.87$ and 0.9 , respectively). HFBP was also weakly correlated with $\mathrm{Cl}_{i}$. The $N_{i}$ was not correlated with any of the applied BPV indices.

There was no significant correlation between blood pressure asymmetry measures and mean RR interval (for the 30-min recording in
Table 2 Results of Spearman correlation between measures of blood pressure asymmetry and continuous clinical variables, or indices of autonomic modulation of the cardiovascular system

\begin{tabular}{|c|c|c|c|c|}
\hline & $S D 1_{i}^{2}$ & $S D 1_{r}^{2}$ & $C 1_{\mathrm{i}}$ & $\mathrm{N}_{\mathrm{i}}$ \\
\hline \multicolumn{5}{|l|}{ Clinical characteristics } \\
\hline Age & $-0.14^{*}$ & $-0.14^{*}$ & 0.08 & $-0.19 * *$ \\
\hline Body mass index & -0.06 & -0.06 & 0.07 & 0.01 \\
\hline Waist-to-hip ratio & -0.07 & -0.07 & 0.06 & -0.04 \\
\hline Height & -0.05 & -0.05 & 0.06 & -0.11 \\
\hline Weight & -0.07 & -0.07 & 0.07 & -0.04 \\
\hline Resting heart rate & 0.07 & 0.07 & -0.11 & 0.10 \\
\hline Brachial systolic blood pressure & 0.11 & $0.14^{*}$ & -0.07 & 0.07 \\
\hline Brachial diastolic blood pressure & 0.07 & 0.10 & $-0.15^{*}$ & $0.22 * * *$ \\
\hline Brachial pulse pressure & 0.05 & 0.05 & 0.07 & -0.12 \\
\hline \multicolumn{5}{|l|}{ SBP time series } \\
\hline Mean SBP & $0.30 * * *$ & $0.33^{* * *}$ & $-0.17^{*}$ & $0.15^{*}$ \\
\hline SDBP & $0.43^{* * *}$ & $0.39 * * *$ & 0.08 & -0.06 \\
\hline LFBP & $0.49 * * *$ & $0.48 * * *$ & 0.11 & -0.10 \\
\hline HFBP & $0.87^{* * *}$ & $0.90 * * *$ & $-0.15^{*}$ & 0.02 \\
\hline \multicolumn{5}{|l|}{$R R$ interval time series } \\
\hline Mean RR interval & -0.07 & -0.08 & 0.11 & -0.13 \\
\hline SDNN & $0.17 * *$ & $0.19 * *$ & -0.06 & 0.02 \\
\hline LFRR & $0.20^{* *}$ & $0.22 * *$ & -0.04 & -0.01 \\
\hline HFRR & 0.12 & $0.15^{*}$ & -0.08 & 0.02 \\
\hline $\mathrm{LFRR}_{\mathrm{nu}}$ & 0.05 & 0.02 & 0.11 & $-0.13^{*}$ \\
\hline LFRR/HFRR & 0.05 & 0.02 & 0.11 & $-0.13^{*}$ \\
\hline $\mathrm{SD} 1 d^{2}$ & $0.14^{*}$ & $0.15^{*}$ & -0.04 & 0.01 \\
\hline $\mathrm{SD} 1_{a}^{2}$ & 0.13 & $0.14^{*}$ & -0.05 & 0.01 \\
\hline $\mathrm{C} 1_{d}$ & 0.08 & 0.10 & 0.02 & -0.01 \\
\hline$N_{d}$ & -0.12 & $-0.14^{*}$ & 0.01 & 0.01 \\
\hline \multicolumn{5}{|l|}{ Baroreflex } \\
\hline BRS & $-0.15^{*}$ & -0.13 & -0.09 & 0.10 \\
\hline Baroreflex delay & -0.09 & -0.12 & 0.08 & -0.11 \\
\hline
\end{tabular}

Abbreviations: BPV, blood pressure variability; BRS, baroreflex sensitivity; $\mathrm{C} 1_{d}$, relative contributions of heart rate decelerations to short-term HRV; $C 1_{i}$, relative contributions of SBP increases to short-term BPV; HFBP, power of high frequency oscillations of SBP; HFRR, power of high frequency oscillations of RR intervals; HRV, heart rate variability; IQR, inter-quartile range; LFBP, power of low frequency oscillations of SBP; LFRR, power of low frequency oscillations of RR intervals; LFRR/HFRR, the ratio of LFRR to HFRR. LFRR power of low frequency oscillations of RR intervals in normalized units; $N_{d}$, the relative number of heart rate decelerations to the number of all changing RR intervals; $N_{i}$, the relative number of SBP increases to the number of all changing values of SBP; RR interval, duration of cardiac cycle of sinus origin; SBP, systolic blood pressure; $\mathrm{SD} 1_{a}^{2}$, the part of short-term HRV derived from heart rate accelerations; $\mathrm{SD} 1 d^{2}$, the part of short-term HRV derived from heart rate decelerations; $\mathrm{SD} 1_{i}^{2}$, the part of SBP short-term variance derived from SBP increases; $\mathrm{SD} 1_{r}^{2}$, the part of SBPshort-term variance derived from SBP reductions; SDBP, the standard deviation of SBP values for all normal beats; SDNN, standard deviation of all normal RR intervals.

${ }^{*} P<0.05 ; * * P<0.01 ; * * * P<0.001$

supine position). The correlations between the values of blood pressure asymmetry descriptors and parameters of HRV, and heart rate asymmetry were weak or not present at all, depending on the analyzed variables.

For baroreflex function, there was only one weak significant correlation between baroreflex sensitivity and $\mathrm{SD} 1_{i}{ }^{2}$ and borderline with $\mathrm{SD}_{r}{ }^{2}(P=0.051)$. Baroreflex delay was not significantly correlated with any measure of blood pressure asymmetry.

\section{Proportions of blood pressure asymmetry and heart rate} asymmetry and their co-existence

The analysis of proportions of blood pressure asymmetry revealed that there were 186 individuals in the studied group with $\mathrm{Cl}_{i}>50 \%$, that 
Table 3 The analysis of co-existence of blood pressure asymmetry $\left(C 1_{i}>50 \%, N_{i}<50 \%\right)$ with heart rate asymmetry $\left(\mathrm{Cl}_{d}>50 \%\right.$, $N_{d}<50 \%$ ) with observed and predicted number of individuals

\begin{tabular}{|c|c|c|c|c|}
\hline & \multicolumn{2}{|c|}{ Observed } & \multicolumn{2}{|c|}{ Predicted } \\
\hline & \multicolumn{2}{|c|}{$C 1_{\mathrm{i}}>50 \%$} & \multicolumn{2}{|c|}{$C 1_{\mathrm{i}}>50 \%$} \\
\hline & False & True & False & True \\
\hline \multicolumn{5}{|c|}{$C 1_{d}>50 \%$} \\
\hline False & 6 & 32 & 6.9 & 31.1 \\
\hline \multirow[t]{2}{*}{ True } & 35 & 154 & 34.1 & 154.9 \\
\hline & \multicolumn{2}{|c|}{$\mathrm{N}_{\mathrm{i}}<50 \%$} & \multicolumn{2}{|c|}{$\mathrm{N}_{\mathrm{i}}<50 \%$} \\
\hline \multicolumn{5}{|c|}{$N_{d}<50 \%$} \\
\hline False & 22 & 66 & 22.1 & 65.9 \\
\hline True & 35 & 104 & 34.9 & 104.1 \\
\hline
\end{tabular}

Abbreviations: $\mathrm{C} 1_{d}$, relative contributions of heart rate decelerations to short-term heart rate variability; $\mathrm{Cl}_{1}$, relative contributions of systolic blood pressure increases to short-term blood pressure variability; $N_{d}$, the relative number of heart rate decelerations to the number of all changing RR intervals; $N_{i}$, the relative number of systolic blood pressure increases to the changing RR intervals; $N_{i}$, the relative number of systolic blood pressure increases to the
number of all changing values of systolic blood pressure; RR interval, duration of cardiac cycle of sinus origin.

is, proportion of $0.82(95 \% \mathrm{CI}: 0.76-0.87 ; P<0.0001)$. There were also 170 subjects with $N_{i}<50 \%$ with proportion of 0.75 (95\% CI: 0.69 $0.80 ; P<0.0001)$. Both of the analyzed proportions were significantly higher than 0.5 , that is, different from a random proportion, which would indicate no blood pressure asymmetry.

Heart rate asymmetry proportions were as follows. There were 189 individuals in the studied group with $\mathrm{Cl}_{d}>50 \%$, that is, proportion of 0.83 (95\% CI: $0.78-0.88 ; P<0.0001$ ). There were also 140 subjects with $N_{d}<50 \%$ with proportion of 0.62 (95\% CI: $0.55-0.68$; $P=0.0005)$. Both of the analyzed proportions were significantly higher than 0.5 , that is, different from a random proportion, which would indicate no heart rate asymmetry.

The proportion analysis of co-existence of both blood pressure asymmetry and heart rate asymmetry revealed that both phenomena are completely independent. The results of proportion analysis of $\mathrm{Cl}_{i}>50 \%$ and $\mathrm{Cl}_{d}>50 \%$ or $N_{i}<50 \%$ and $N_{d}<50 \%$ presented in Table 3 are a textbook example of independence. The expected numbers are the products of respective marginal probabilities multiplied by the sample size. As can be seen from the table, the results are not only close-they are right on target.

\section{Relation of blood pressure asymmetry to gender}

The Mann-Whitney $U$ test showed no gender differences in the values of blood pressure asymmetry indices (Table 4). The binomial test revealed that the proportion of women with $C_{i}>50 \%(0.81 ; 95 \%$ CI: $0.72-0.89)$ and $N_{i}<50 \%(0.71 ; 95 \%$ CI: $0.61-0.80)$ was significantly higher than 0.5 ( $P<0.0001$ for both). Similarly, the proportion of men with $C_{i}>50 \%(0.82$; 95\% CI: $0.75-0.88)$ and $N_{i}<50 \%(0.78$; 95\% CI: $0.70-0.85)$ was significantly higher than 0.5 ( $P<0.0001$ for both). In addition, the Fisher's exact test revealed no gender difference in the presence of blood pressure asymmetry both for $C_{i}>50 \%$ and $N_{i}<50 \%$.

\section{DISCUSSION}

With this study we show that SBP increases are less numerous, but they have a significantly larger contribution to short-term BPV compared with SBP reductions in 30-min resting recordings in healthy
Table 4 Comparison of values of blood pressure asymmetry indices between studied women and men

\begin{tabular}{|c|c|c|c|c|c|}
\hline & \multicolumn{2}{|c|}{ Women } & \multirow[b]{2}{*}{ P-value } & \multicolumn{2}{|c|}{ Men } \\
\hline & Median & $I Q R$ & & Median & $I Q R$ \\
\hline$C_{i}$ & 52.5 & $50.4-54.1$ & NS & 53.1 & $51.1-55.3$ \\
\hline$C_{d}$ & 47.5 & $45.9-49.6$ & NS & 46.9 & $44.7-48.9$ \\
\hline$N_{i}$ & 49.1 & $47.8-50.3$ & NS & 48.7 & $46.9-49.8$ \\
\hline$N_{d}$ & 51.0 & $49.7-52.2$ & NS & 51.3 & $50.2-53.1$ \\
\hline $\mathrm{SD} 1 i^{2}$ & 1.5 & $1.2-2.0$ & NS & 1.5 & $1.3-1.9$ \\
\hline $\mathrm{SD} 1 d^{2}$ & 1.4 & $1.2-1.8$ & NS & 1.4 & $1.2-1.8$ \\
\hline
\end{tabular}

Abbreviations: $C_{i}$, median contribution of systolic blood pressure increases; $C_{d}$, median contribution of systolic blood pressure decreases; IQR, inter-quartile range, $N_{d}$, the relative number of heart rate decelerations to the number of all changing RR intervals, $N_{i}$, the relative number of systolic blood pressure increases to the number of all changing values of systolic blood pressure, NS, not significant; RR interval, duration of cardiac cycle of sinus origin; $\mathrm{SD} 1{ }^{2}$, the part of short-term heart rate variability derived from heart rate decelerations; $\mathrm{SD} 1_{l}^{2}$, the part of SBP short-term variance derived from systolic blood preessure increases.

young people at supine position. The unequal contributions of SBP increases and reductions to the short-term variance of SBP clearly shows that BPV is asymmetric and for this reason we call this phenomenon blood pressure asymmetry. The frequency of blood pressure asymmetry is significantly different from 0.5 for both proposed measures of blood pressure asymmetry, that is, $\mathrm{Cl}_{i}$ and $N_{i}$, it prevails in an overwhelming majority (at least 75\%) of the studied participants. Further, it appears that the measures of blood pressure asymmetry are weakly correlated with age and brachial blood pressure, as well as various indirect descriptors of autonomic modulation of the cardiovascular system (selected parameters of BPV, HRV, heart rate asymmetry and baroreflex sensitivity). The analysis of a potential interaction between blood pressure asymmetry and heart rate asymmetry shows that both phenomena are independent. Finally, there is no gender difference both in the expression and frequency of blood pressure asymmetry.

The study was performed at supine rest with no provocations or autonomic maneuvers. Such recording conditions have influenced both the cardiovascular and the autonomic nervous systems, and also the results of BPV, HRV, heart rate asymmetry and baroreflex function. For this reason we must limit further discussion and conclusions to resting conditions.

Bidirectional changes in SBP, that is, SBP increases and reductions, determine BPV. These changes are unequal in both magnitude and number-less numerous SBP increases are accompanied by larger variance compared with SBP reductions. A change in SBP between two consecutive beats is bigger for SBP increases than reductions, and it might be speculated that some controlling mechanisms should compensate this difference by decreasing the number of SBP increases. In fact, this number is significantly lower than the number of SBP reductions, however the compensatory effect does not seem to be fully working, and thus we observe blood pressure asymmetry.

The phenomenon of blood pressure asymmetry reported here is present in over $80 \%$ of healthy young individuals, when quantified by the magnitude of SBP changes, that is, $C_{i} v s . \mathrm{C}_{p}$ or in $75 \%$ of subjects when measured by the relative number of SBP changes, that is, $N_{i} v s$. $N_{r}$. These figures clearly show that blood pressure asymmetry is a consistent finding. The use of exactly the same sets of SBP time series, but with shuffled order of consecutive values (data not shown) causes disappearance of blood pressure asymmetry. The shuffling process, by a complete destruction of the natural structure of the SBP signal, equalizes the numbers of SBP increases and reductions and their 
contribution to short-term BPV. Because blood pressure asymmetry is present only in recordings with preserved natural order of consecutive SBP values, we conclude that this phenomenon is related to the structure of such recordings.

The expression of blood pressure asymmetry is quite similar in men and women, it is not related to anthropometrics like body height and weight, body mass index or waist-to-hip ratio. On the other hand, there are significant, although weak, correlations between blood pressure asymmetry and age or brachial blood pressure. It should, however, be kept in mind that median values of anthropometric measures correspond to normal values, range of age of the studied participants is quite narrow (12 years only) and brachial blood pressure was $<140 / 90 \mathrm{~mm} \mathrm{Hg}$.

Blood pressure asymmetry is another example of asymmetric feature of the cardiovascular function, the first being the heart rate asymmetry. ${ }^{12,16}$ Blood pressure changes trigger heart rate alterations, and thus it might be concluded that the heart rate asymmetry is an effect of blood pressure asymmetry. However, to our surprise, both phenomena of heart rate asymmetry and blood pressure asymmetry were very weakly correlated (Table 2) with no relation between their coexistence (Table 3 ). Heart rate changes induced by blood pressure are mediated by baroreflex, which has also asymmetrical properties. ${ }^{1,3-7,9,14,16,18-21,27,31}$ The baroreflex sensitivity is different for blood pressure increases and reductions, which is evident by comparing phenylephrine with nitroprusside infusion, or neck pressure with neck suction methods. ${ }^{18-20}$ Heart rate asymmetry, although independent of blood pressure asymmetry, is probably related to baroreflex, which is supported by the observation that heart rate asymmetry is correlated with measures of baroreflex function. ${ }^{32}$

Porta et al. ${ }^{33}$ showed that asymmetrical features of HRV change during sympathetic provocation like head-up tilting and that heart rate asymmetry expression increases during the day compared with night, both in healthy people and patients with congestive heart failure. ${ }^{17,24}$ Recently, we showed that heart rate asymmetry is reduced in patients with type 1 diabetes of at least 25-year duration of the disease both in 10-min resting and in 24-h ambulatory Holter ECG recordings. These investigations show that heart rate asymmetry is a dynamic phenomenon and is related to the sympathetic-parasympathetic balance.

The current physiological study suggests that blood pressure asymmetry is under some autonomic control, as it is significantly correlated with indices of HRV, BPV and baroreflex function. One group of these correlations is of particular interest, that is, the correlation between $\mathrm{SD} 1_{i}^{2}$ or $\mathrm{SD} 1_{r}^{2}$ and $\mathrm{BPV}$. The values of $\mathrm{SD} 1_{i}^{2}$ or $\mathrm{SD} 1_{r}{ }^{2}$ are moderately correlated with LFBP but strongly with HFBP. LFBP represents low frequency sympathetic oscillations causing fluctuations in vasomotor tone and systemic vascular resistance whereas HFBP is assumed to represent mainly mechanical effects of breathing on the cardiovascular system or vagal effects on heart rate and cardiac output. ${ }^{3-6,9,27,34}$ If so, then it might be plausible that blood pressure asymmetry is a net effect of the changes in sympathetic tone, respiratory influences and the amount of circulating blood. However, we are aware that this is a speculation only and that the mechanisms responsible for systolic blood pressure asymmetry need thorough investigations. There is also one possibility that the observed strong correlations between $\mathrm{SD}_{i}{ }^{2}, \mathrm{SD}_{r}{ }^{2}$ and HFBP is a result of mutual mathematical dependence of these variables. ${ }^{1}$ Both $\mathrm{SD}_{i}{ }^{2}$ and $\mathrm{SD}_{r}{ }^{2}$ are rescaled derivatives of root mean square of successive differences in SBP values between consecutive heart beats, which, due to mathematical relations, is very strongly correlated with the HFBP. ${ }^{1,11}$
Limitations of the study must be recognized. Only data from young (aged 19-31 years old), healthy people were analyzed, so the results should not be extrapolated to children, as well as older adults. The recordings were collected at supine rest with no maneuvers changing sympathetic-parasympathetic balance. Therefore, it is unknown whether blood pressure asymmetry can be observed in non-resting conditions like physical activity, in an upright position, squatting, slow breathing or even during sleep with its different phases, that is, rapid eye movement and non-rapid eye movement. We have also used non-invasive measurement of finger blood pressure and it has been shown that the LFBP can be overestimated by such recordings. ${ }^{35,36}$ Whether blood pressure asymmetry is still present when non-invasive measurements are replaced by invasive ones is unclear. However, in most physiological and clinical studies continuous noninvasive recordings of blood pressure signal at the finger level are most commonly used. ${ }^{4-9,14,18-21,27,35}$

Studies on blood pressure asymmetry can develop in different directions. First, the explanation of the mechanisms responsible for this phenomenon like the influence of respiration, autonomic provocations or blockade is necessary. Second, a more detailed analysis of the relation of blood pressure asymmetry to age, arterial stiffness, endothelial function, other hemodynamic parameters like stroke volume, cardiac output, vascular resistance and so on might be helpful in better understanding of this phenomenon, its origin and physiological importance. Third, clinical studies like the analysis of blood pressure asymmetry in healthy subjects and patients with hypotension, hypertension, or changes caused by specific pharmacological treatment or interventions on carotid arteries or aorta. The evaluation of blood pressure asymmetry in patients with cardiovascular risk factors (for example, diabetes, smoking), diseases (for example, post-infarction, heart failure) and complications (for example, left ventricular hypertrophy, stroke or end-stage renal disease) is another important clinical topic.

This study shows the presence of blood pressure asymmetry in young, healthy people at rest. It is manifested by a significantly larger contribution to short-term SBP variability from SBP increases than reductions, and a significantly smaller number of SBP increases than reductions.

The clinical value of blood pressure asymmetry has not been evaluated yet, however, one immediate consequence of the presence of this phenomenon is for physiological and theoretical models involving blood pressure and its variability. The presence of blood pressure asymmetry in such models should be considered and possibly incorporated.

\section{CONFLICT OF INTEREST}

The authors declare no conflict of interest.

\section{ACKNOWLEDGEMENTS}

This study was supported by University of Medical Sciences in Poznan Internal Funding Sources.

1 Task Force of the European Society of Cardiology and the North American Society of Pacing and Electrophysiology. Heart rate variability: standards of measurement, physiological interpretation, and clinical use. Circulation 1996; 93: 1043-1065.

2 Kleiger RE, Stein PK, Bigger Jr JT. Heart rate variability: measurement and clinical utility. Ann Noninvasive Electrocardiol 2005; 10: 88-101.

3 Mancia G, Parati G, Casadei R, Di Rienzo M, Zanchetti A. Arterial baroreflexes and blood pressure and heart rate variabilities in humans. Hypertension 1986; 8: 147-153.

4 Julien C, Malpas SC, Stauss HM. Sympathetic modulation of blood pressure variability. J Hypertens 2001; 19: 1707-1712. 
5 Aono T, Sato T, Nishinaga M, Kawamoto A, Ozawa T. Power spectral analysis of spontaneous blood pressure and heart rate variability in elderly hypertensives. Hypertens Res 1996; 19: 9-16.

6 Laitinen T, Hartikainen J, Niskanen L, Geelen G, Länsimies E. Sympathovagal balance is major determinant of short-term blood pressure variability in healthy subjects. $A m \mathrm{~J}$ Physiol Heart Circ Physiol 1999; 276: H1245-H1252.

7 Hamner JW, Morin RJ, Rudolph JL, Taylor JA. Inconsistent link between low-frequency oscillations: R-R interval responses to augmented Mayer waves. J Appl Physiol 2001; 90: 1559-1564.

8 Taylor JA, Williams TD, Seals DR, Davy KP. Low-frequency arterial pressure fluctuations do not reflect sympathetic outflow: gender and age differences. Am J Physiol Heart Circ Physiol 1998; 274: H1194-H1201.

9 Parati G, Saul JP, Di Rienzo M, Mancia G. Spectral analysis of blood pressure and heart rate variability in evaluating cardiovascular regulation. A critical appraisal. Hypertension 1995; 25: 1276-1286.

10 Brennan M, Palaniswami M, Kamen P. Poincaré plot interpretation using a physiological model of HRV based on a network of oscillators. Am J Physiol Heart Circ Physiol 2002; 283: H1873-H1886.

11 Brennan M, Palaniswami M, Kamen P. Do existing measures of Poincaré plot geometry reflect nonlinear features of heart rate variability? IEEE Trans Biomed Eng 2001; 48: 1342-1347.

12 Piskorski J, Guzik P. Geometry of the Poincaré plot of RR intervals and its asymmetry in healthy adults. Physiol Meas 2007; 28: 287-300.

13 Blanc J, Ponchon P, Laude D, Elghozi JL, Jover B. Blood pressure variability in established L-NAME hypertension in rats. J Hypertens 1999; 17: 1527-1534.

14 Westerhof BE, Gisolf J, Stok WJ, Wesseling KH, Karemaker JM. Time-domain crosscorrelation baroreflex sensitivity: performance on the EUROBAVAR data set. J Hypertens 2004: 22: 1371-1380.

15 Bauer A, Kantelhardt JW, Barthel P, Schneider R, Mäkikallio T, UIm K, Hnatkova K, Schömig A, Huikuri H, Bunde A, Malik M, Schmidt G. Deceleration capacity of heart rate as a predictor of mortality after myocardial infarction: cohort study. Lancet 2006; 367: 1674-1681.

16 Guzik P, Piskorski J, Krauze T, Wykretowicz A, Wysocki H. Heart rate asymmetry by Poincaré plots of RR intervals. Biomed Tech (Berl) 2006; 51: 272-275.

17 Porta A, Casali KR, Casali AG, Gnecchi-Ruscone T, Tobaldini E, Montano N, Lange S, Geue D, Cysarz D, Van Leeuwen P. Temporal asymmetries of short-term heart period variability are linked to autonomic regulation. Am J Physiol Regul Integr Comp Physiol 2008; 295: R550-R557.

18 Rudas L, Crossman AA, Morillo CA, Halliwill JR, Tahvanainen KU, Kuusela TA, Eckberg DL. Human sympathetic and vagal baroreflex responses to sequential nitroprusside and phenylephrine. Am J Physiol Heart Circ Physiol 1999; 276: $\mathrm{H} 1691-\mathrm{H} 1698$.

19 Shepherd AM, Lin MS, McNay JL, Musgrave GE, Keeton TK. Baroreflex sensitivity modulates vasodepressor response to nitroprusside. Hypertension 1983; 5: 79-85.

20 Parlow J, Viale JP, Annat G, Hughson R, Quintin L. Spontaneous cardiac baroreflex in humans. Comparison with drug-induced responses. Hypertension 1995; 25: 1058-1068
21 Eckberg DL. Nonlinearities of the human carotid baroreceptor-cardiac reflex. Circ Res 1980; 47: 208-216.

22 Schneider R, Barthel P, Bauer A, Schmidt G. libRASCH—A Programming Framework for Signal Handling. Comput Cardiol 2004; 31: 53-56.

23 Tulppo MP, Mäkikallio TH, Seppänen T, Laukkanen RT, Huikuri HV. Vagal modulation of heart rate during exercise: effects of age and physical fitness. Am J Physiol Heart Circ Physiol 1998; 274: H424-H429.

24 Porta A, D'addio G, Bassani T, Maestri R, Pinna GD. Assessment of cardiovascular regulation through irreversibility analysis of heart period variability: a $24 \mathrm{~h}$ Holter study in healthy and chronic heart failure populations. Phil Trans Royal Soc A 2009; 367: 1359-1375.

25 Karmakar CK, Khandoker AH, Gubbi J, Palaniswami M. Defining asymmetry in heart rate variability signals using a Poincaré plot. Physiol Meas 2009; 30: 1227-1240.

26 Houa F, Zhuanga J, Biana C, Tonga T, Chena Y, Yina J, Qiuc X, Ninga X. Analysis of heartbeat asymmetry based on multi-scale time irreversibility test. Physica A 2010; 389: 754-760.

27 Castiglioni P, Di Rienzo M, Veicsteinas A, Parati G, Merati G. Mechanisms of blood pressure and heart rate variability: an insight from low-level paraplegia. Am J Physiol Regul Integr Comp Physiol 2007; 292: R1502-R1509.

28 Lomb NR. Least-squares frequency analysis of unequally spaced data. Astrophys Space Sci 1976; 39: 447-462.

29 Laguna P, Moody GB, Mark RG. Power spectral density of unevenly sampled data by least-square analysis: Performance and application to heart rate signals. IEEE Trans Biomed Eng 1998; 45: 698-715.

30 Piskorski J, Guzik P, Krauze T, Zurek S. Cardiopulmonary resonance at $0.1 \mathrm{~Hz}$ demonstrated by averaged Lomb-Scargle periodogram. Cent Eur J Phys 2010; 8: 386-392.

31 Bauer A, Malik M, Schmidt G, Barthel P, Bonnemeier H, Cygankiewicz I, Guzik P, Lombardi F, Müller A, Oto A, Schneider R, Watanabe M, Wichterle D, Zareba W. Heart Rate Turbulence: Standards of measurement, physiological interpretation, and clinical use International Society for Holter and Noninvasive Electrophysiology Consensus. J Am Coll Cardiol 2008; 52: 1353-1365.

32 Guzik P, Piskorski J. Asymmetric properties of short-, long-term and total heart rate variability. Proceedings of the 6th Conference of the European Study Group on Cardiovascular Oscillations. 12-14.04. 2010. Berlin, Germany: P083:1-3.

33 Guzik P, Piskorski J, Contreras P, Migliaro ER. Asymmetrical properties of heart rate variability in type 1 diabetes. Clin Auton Res 2010; 20: 255-257.

34 Saul JP, Berger RD, Albrecht P, Stein SP, Chen MH, Cohen RJ. Transfer function analysis of the circulation: unique insights into cardiovascular regulation. Am J Physiol Heart Circ Physiol 1991; 261: H1231-H1245

35 Parati G, Di Rienzo M, Bertinieri G, Pomidossi G, Casadei R, Groppelli A, Pedotti A, Zanchetti A, Mancia G. Evaluation of the baroreceptor-heart rate reflex by 24-h intra-arterial blood pressure monitoring in humans. Hypertension 1988; 12 : 214-222.

36 Omboni S, Parati G, Frattola A, Mutti E, Di Rienzo M, Castiglioni P, Mancia G. Spectral and sequence analysis of finger blood pressure variability. Comparison with analysis of intra-arterial recordings. Hypertension 1993; 22: 26-33. 